\title{
Abscissas and Weights for Lobatto Quadrature of High Order
}

\section{By Philip Rabinowitz}

In recent years, Gaussian quadrature has become the standard method for numerical integration in many computer installations [1, 2]. In general, Gaussian rules are most economical since an $n$-point rule is exact for polynomials up to degree $2 n-1$ and no rule can do better. However, for particular classes of functions and for particular applications, other rules may be more efficient. Thus there are occasions when we prefer a closed rule, i.e. one which includes among its abscissas the two end points of the integration interval. This is the case when the integrand vanishes at the two end points as it does in Longman's method for evaluating integrals of oscillating functions [3]. If we want to check a quadrature over a given interval by doing two additional quadratures, each over half the interval, then a closed rule will save at least two evaluations of the integrand.

Let us normalize our integration interval to $(-1,1)$ and consider the closed symmetric $n$-point integration rule with $n$ odd, $n=2 m+1$,

$$
\int_{1}^{1} f(x) d x=\sum_{k=-m}^{m} a_{k n} f\left(x_{k n}\right)+E_{n}
$$

with $x_{ \pm m n}= \pm 1, x_{-k n}=-x_{k n}$, and $a_{-k n}=a_{k n}$. It was first shown by Lobatto* [4] (cf [5]) that the $2 n-2$ quantities, $n-2$ abscissas $x_{k n}$ and $n$ weights $a_{k n}$, can be so chosen that the integration is exact for polynomials up to degree $2 n-3$. The abscissas $x_{k n}$ are the zeros of $P_{n-1}^{\prime}(x)$, the first derivative of the Legendre polynomial of degree $n-1, P_{n-1}(x)$, and the corresponding weights $a_{k n}$ are given by

$$
a_{k n}=\frac{2}{n(n-1)\left[P_{n-1}\left(x_{k n}\right)\right]^{2}}
$$

while $a_{m n}=a_{-m n}=2 / n(n-1)$. In this case

$$
E_{n}=-\frac{n(n-1)^{3} 2^{2 n-1}[(n-2) !]^{4}}{(2 n-1)[(2 n-2) !]^{3}} f^{(2 n-2)}(\xi) \text {. }
$$

Tables of abscissas and weights for Lobatto quadrature (where only the values of $k=0, \cdots, m$ are listed) were calculated on WEIZAC to 19D for $n=5(4)$

Received May 20, 1959; in revised form August 7, 1959.

* According to Scarborough [6], Lobatto modified Gauss's integration rule so as to include the end values and also the value of the function at the midpoint of the interval. Radau [7] extended Lobatto's method to the general case of $m$ fixed abscissas in an $n$-point rule $(m \leqq n)$. Kopal [8] designates by Radau quadrature all rules with $m$ preassigned abscissas. However, Hildebrand [5] and others use Radau quadrature to denote the case where only the left-hand endpoint of the integration interval is preassigned and Lobatto quadrature, the case where both endpoints are preassigned. Radau [7] tabulated Lobatto abscissas and weights for $n=$ 2(1)8 to eight decimal places and for $n=9(1) 11$ to ten decimal places and Kopal [8] has reproduced them. 
25(8) 49(16) 97. Table 1 lists these values, with the exception of $n=81,97$. Values for $n=81,97$ have been deposited in the Unpublished Mathematical Tables File. The zeros of $P_{n-1}^{\prime}(x)$ were computed with double precision routines using Newton's method,

TABLE 1

Abscissas and weights for Lobatto quadrature

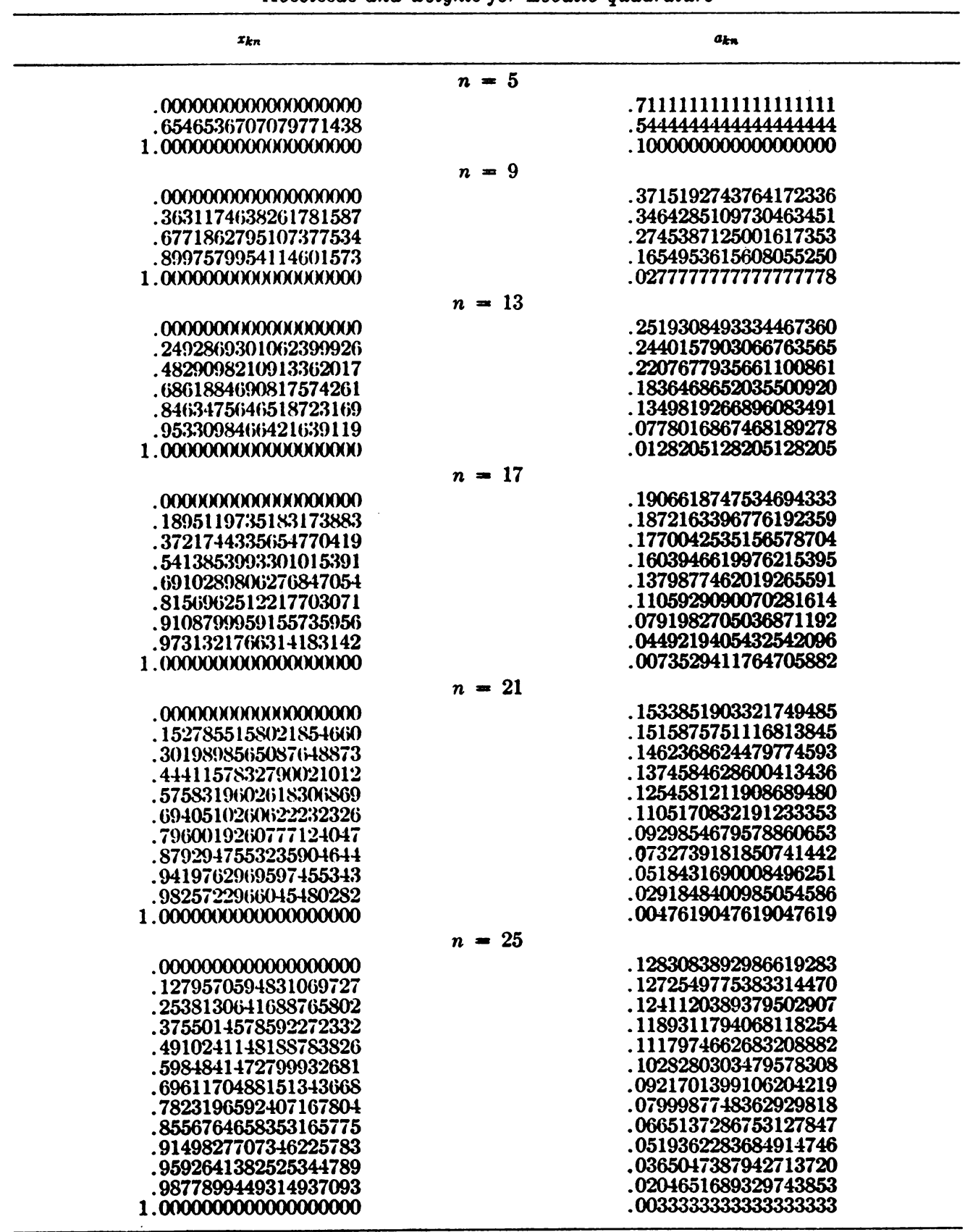


Table 1-Continued

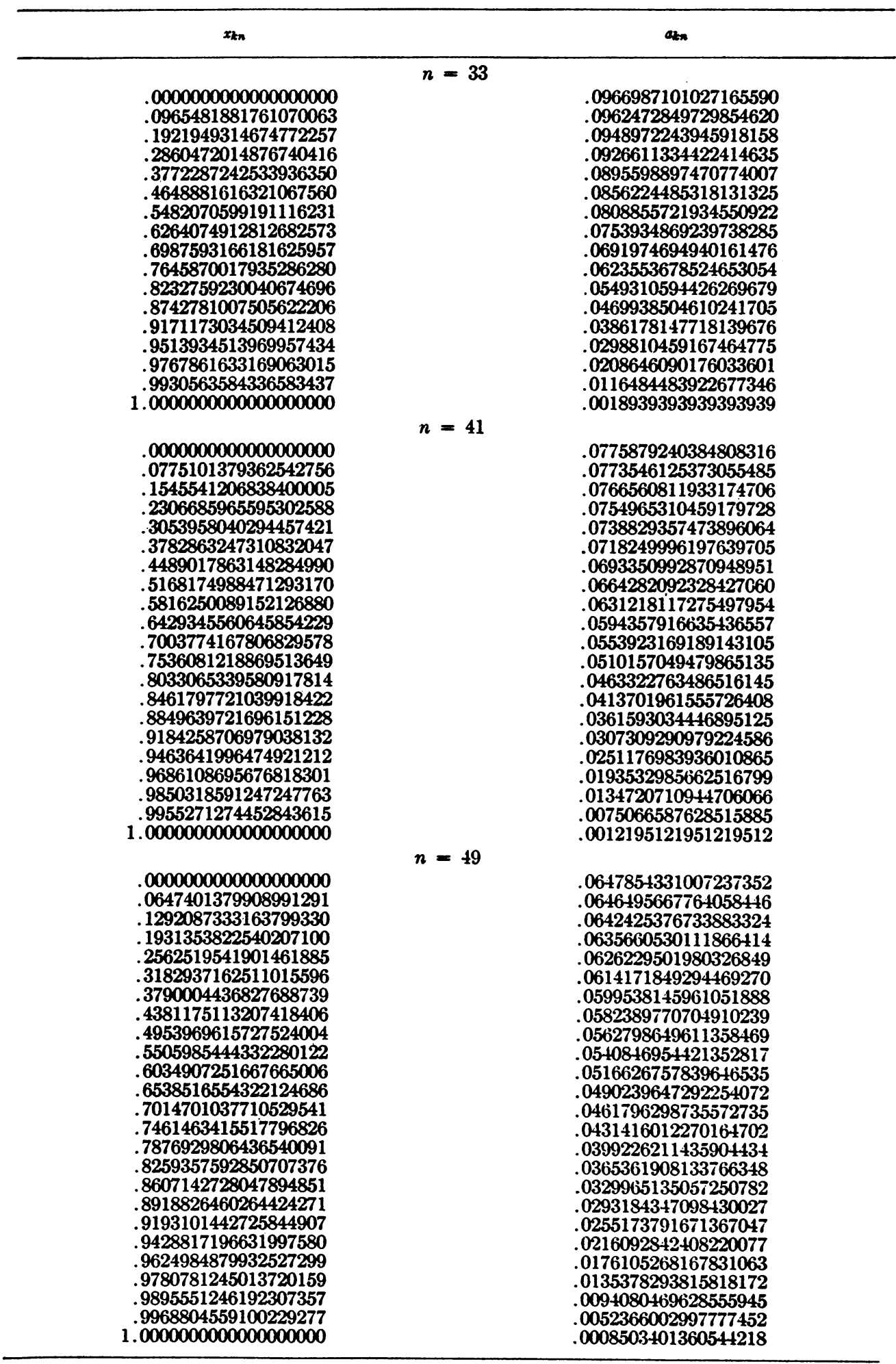


TABLE 1-Continued

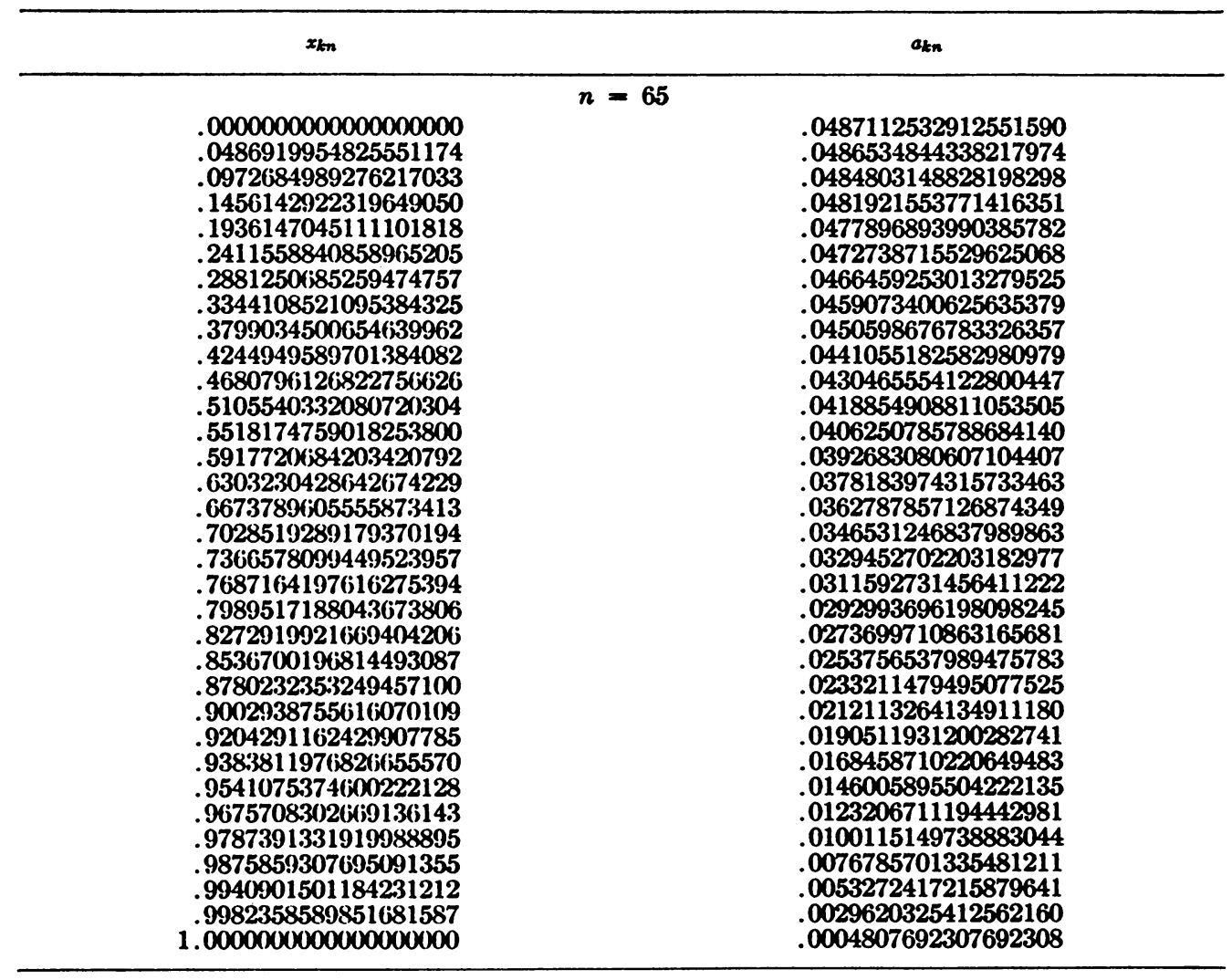

$$
x_{k n}^{(i+1)}=x_{k n}^{(i)}-\frac{P_{n-1}^{\prime}\left(x_{k n}^{(i)}\right)}{P_{n-1}^{\prime \prime}\left(x_{k n}^{(i)}\right)}=x_{k n}^{(i)}-\Delta .
$$

Since [9]

$$
\left(1-x^{2}\right) P_{n}^{\prime}(x)=n\left[P_{n-1}(x)-x P_{n}(x)\right]
$$

and

$$
\left(1-x^{2}\right) P_{n}^{\prime \prime}(x)=2 x P_{n}^{\prime}(x)-n(n+1) P_{n}(x),
$$

$\Delta$ reduced to the following formula which only involves Legendre polynomials:

$$
\Delta=\frac{P_{n-2}\left(x_{k n}^{(i)}\right)-x_{k n}^{(i)} P_{n-1}\left(x_{k n}^{(i)}\right)}{2 x_{k n}^{(i)}\left[P_{n-2}\left(x_{k n}^{(i j}\right)-x_{k n}^{(i j} P_{n-1}\left(x_{k n}^{(i)}\right)\right]-n P_{n-1}\left(x_{k n}^{(i)}\right)} .
$$

The iteration was terminated when $|\Delta|$ became smaller than $2^{-74}$. The initial values $x_{k n}^{(0)}$ were taken to be the arithmetic means of two successive zeros of $P_{n-1}(x)$ tabulated by Davis and Rabinowitz. [10,11] With this choice of initial value, convergence was achieved in at most six iterations.

The values in the table were checked on the computer by calculating the sums $\sum_{k-m}^{m} a_{k n} x_{k n}^{r}$ for $r=0,2,4,8,16,32$. For $n$ such that $r \leqq 2 n-3$, these sums were equal to $(2 / r+1)$ to 19 decimal places. In addition $\sum_{k=-m}^{m} a_{k n}$ was computed 
by hand from the final manuscript and was found to deviate from the correct value 2 by at most 4 units in the 19th decimal place. This accuracy is acceptable since we were summing rounded numbers. A further check was made on the computer by calculating $\sum_{k=1}^{m} x_{k n}^{2}$ and $\prod_{k=1}^{m} x_{k n}$. Each sum checked to 19 decimal places with the true value $(n-2)(n-3) / 2(2 n-3)$ while the square of each product checked to at least ten significant figures with the true value

$$
\frac{\left(\begin{array}{l}
n+1 \\
m+1
\end{array}\right)(m+1)}{2\left(\begin{array}{c}
2 n-2 \\
n-1
\end{array}\right)}
$$

These two identities are derived as follows:

$$
\begin{aligned}
P_{n-1}(x) & =2^{-(n-1)} \sum_{t=0}^{m}(-1)^{t}\left(\begin{array}{c}
n-1 \\
t
\end{array}\right)\left(\begin{array}{c}
2 n-2-2 t \\
n-1
\end{array}\right) x^{n-1-2 t} \\
P_{n-1}^{\prime}(x) & =2^{-(n-1)} \sum_{t=0}^{m}(-1)^{t}\left(\begin{array}{c}
n-1 \\
t
\end{array}\right)\left(\begin{array}{c}
2 n-2-2 t \\
n-1
\end{array}\right)(n-1-2 t) x^{n-2-2 t} \\
& =2^{-(n-1)} x \sum_{t=0}^{m-1}(-1)^{t}\left(\begin{array}{c}
n-1 \\
t
\end{array}\right)\left(\begin{array}{c}
2 n-2-2 t \\
n-1
\end{array}\right)(n-1-2 t)\left(x^{2}\right)^{m-t-1} \\
& =x\left[a_{0}\left(x^{2}\right)^{m-1}+a_{1}\left(x^{2}\right)^{m-2}+\cdots+a_{m-2} x^{2}+a_{m-1}\right] \\
& =a_{0} x \prod_{k=1}^{m}\left(x+x_{k n}\right)\left(x-x_{k n}\right)=a_{0} x \prod_{k=1}^{m}\left(x^{2}-x_{k n}^{2}\right) .
\end{aligned}
$$

Hence

$$
\sum_{k=1}^{m} \dot{x}^{2}=-\frac{a_{1}}{a_{0}}=\frac{\left(\begin{array}{c}
n-1 \\
1
\end{array}\right)\left(\begin{array}{c}
2 n-4 \\
n-1
\end{array}\right)(n-3)}{\left(\begin{array}{c}
n-1 \\
0
\end{array}\right)\left(\begin{array}{c}
2 n-2 \\
n-1
\end{array}\right)(n-1)}=\frac{(n-2)(n-3)}{2(2 n-3)}
$$

and

$$
\prod_{k=1}^{m} x^{2}=(-1)^{m-1} \frac{a_{m-1}}{a_{0}}=\frac{\left(\begin{array}{c}
n-1 \\
m-1
\end{array}\right)\left(\begin{array}{c}
2 n-2 m \\
n-1
\end{array}\right) 2}{\left(\begin{array}{c}
n-1 \\
0
\end{array}\right)\left(\begin{array}{c}
2 n-2 \\
n-1
\end{array}\right)(n-1)}=\frac{m+1}{2} \frac{\left(\begin{array}{c}
n+1 \\
m+1
\end{array}\right)}{\left(\begin{array}{c}
2 n-2 \\
n-1
\end{array}\right)}
$$

Department of Applied Mathematics

Weizmann Institute of Science

Rehovot, Israel

1. M. W. Wilkes, D. J. Wheeler, \& S. Gile, Programs for an Electronic Digital Computer, 2nd Edition, Addison-Wesley Publishing Co., Inc., Reading, Massachusetts, 1957, p. 86.

2. W. L. FRANK, "Mathematical subroutines for the UNIVAC scientific computer," Computers and Automation, v. 6, No. 9, 1957, p. 10-13.

3. I. M. LoNGMAN, "Note on a method for computing infinite integrals of oscillatory functions," Cambridge Phil. Soc., Proc., v. 52, 1956, p. 764.

4. R. LoBatTo, Lessen over de Integraal-Rekening, The Hague, 1852, p. 207-210.

5. F. B. HILDEBRAND, Introduction to Numerical Analysis, McGraw-Hill Book Co., New York, 1956, p. 334

6. J. B. Scarborough, Numerical Mathematical Analysis, The Johns Hopkins Press, Baltimore, Maryland, 1950, p. 152. 
7. R. RADAO, "Wtude sur les formules d'approximation qui servent a calculer la valeur d'une integrale definie," J. Math. Pures Appl., v. 6, No. 3, 1880, p. 283.

8. Z. Kopal, Numerical Analysis, John Wiley \& Sons, Inc., New York, 1955, p. 390.

9. A. ERDELI, wT AL., Higher Transcendental Functions, v. 2, McGraw-Hill Book Co., New York, 1953 p. 179.

10. P. DAvis \& P. RABinowitz, "Abscissas and weights for Gaussian quadratures of high order," NBS, Jn. of Research, v. 56, 1956, p. 35.

11. P. DAvis \& P. RABINowitz, "Additional abscissas and weights for Gaussian quadratures of high order: values for $n=64,80$ and 96 ," NBS, Jn. of Research, v. 60, 1958, p. 618 . 